\title{
Expression and Function of MicroRNAs Encoded by Kaposi's Sarcoma-associated Herpesvirus
}

\author{
E. Gottwein, X. CAi, And B.R. Cullen \\ Center for Virology and Department of Molecular Genetics and Microbiology, Duke University Medical Center, \\ Durham, North Carolina 27710
}

\begin{abstract}
microRNAs (miRNAs) are widely used by animal and plant cells to posttranscriptionally regulate cellular gene expression, but this regulatory mechanism can also be used by pathogenic viruses for the same purpose. It is now well established that numerous miRNAs are expressed by a wide range of pathogenic herpesviruses, although their mRNA targets and role in the viral life cycle remain unclear. Here, we discuss what is currently known about the expression and function of the 12 miRNAs that are expressed by the pathogenic gamma herpesvirus Kaposi's sarcoma-associated herpesvirus in latently infected human B cells.
\end{abstract}

miRNAs are a class of noncoding RNAs, generally 21 or 22 nucleotides in length, that are expressed by all animals and plants examined thus far. More than 300 miRNAs have been identified in human cells, and comparable numbers are likely to be expressed in all other vertebrate animals (Bartel 2004).

Cellular miRNAs are initially transcribed as part of one arm of an approximately 80-nucleotide stem-loop that in turn forms part of a longer capped, polyadenylated RNA (Cai et al. 2004; Lee et al. 2004). This primary miRNA (pri-miRNA) transcript is recognized by the nuclear microprocessor complex, consisting minimally of the RNase III enzyme Drosha and the RNAbinding cofactor DGCR8, which cleaves the stem of the pri-miRNA to liberate an approximately 60-nucleotide RNA hairpin - the pre-miRNA intermediate - bearing a 2-nucleotide 3' overhang (Denli et al. 2004; Gregory et al. 2004; Han et al. 2004). Because the pri-miRNA sequences that flank the pre-miRNA hairpin are retained in the nucleus and degraded, active pri-miRNAs are not capable of simultaneously functioning as an mRNA (Cai et al. 2004). As a result, cellular miRNA hairpins are generally found in noncoding RNAs or within the introns of coding or noncoding RNA polymerase II transcripts (Bartel 2004). However, pri-miRNAs can function as mRNAs if they can be exported to the cytoplasm before they are cleaved by the microprocessor complex (Cai et al. 2004).

The pre-miRNA is exported to the cytoplasm by the karyopherin family member Exportin 5 (Yi et al. 2003), where it is recognized and bound by a second RNase III family member, Dicer. Dicer removes the terminal loop and gives rise to the miRNA duplex intermediate, an approximately 19-bp-long double-stranded RNA (dsRNA) bearing 2-nucleotide $3^{\prime}$ overhangs at each end (Cullen 2004). One strand of this duplex is then selectively incorporated into the RNA-induced silencing complex (RISC), where it acts as a guide RNA to direct RISC to complementary RNA sequences (Hammond et al. 2000; Martinez et al. 2002; Schwarz et al. 2002). Once bound, RISC can inhibit mRNA function by direct cleavage of the mRNA if the degree of complementarity is high or by inhibiting the translation of the mRNA if the degree of complementarity is more limited (Doench et al. 2003; Zeng et al. 2003).

The miRNA-mediated pathway of posttranscriptional gene regulation is thought to have a key role in many aspects of differentiation and development and is active in all cell types at all stages in an animal's development (Bartel 2004). Because miRNA processing can occur efficiently with pri-miRNA precursors as short as about 200 nucleotides (Zeng and Cullen 2003), and because miRNAs are not likely to be antigenic, they also have the potential to provide a facile mechanism for viruses to down-regulate host-cell gene products that might act to limit viral replication. However, as discussed in more detail elsewhere (Cullen 2006), the fact that the primiRNA precursor is cleaved in the nucleus to liberate the pre-miRNA hairpin intermediate, whereas the RNA sequences flanking this hairpin structure are retained in the nucleus and degraded, suggests that viruses which have an RNA genome and/or which replicate in the cytoplasm would be unlikely to encode miRNAs. Similarly, because miRNAs act at the mRNA level, not the protein level, it seems likely that miRNAs would be most advantageous to viruses that establish long-term persistent or latent infections, during which the preexisting pool of target protein could decay, rather than viruses that have short, lytic replication cycles, which might be over before the miRNA-mediated knockdown of an mRNA would have had much effect on the level of the encoded protein. On the basis of this reasoning, it has previously been argued (Cullen 2006) that miRNAs might be prevalent in nuclear DNA viruses that establish long-term latent infections (e.g., herpesviruses), less common in nuclear DNA viruses that undergo exclusively lytic replication cycles (e.g., adenoviruses and polyomaviruses), and rare or absent in cytoplasmic DNA viruses (e.g., poxviruses) or RNA viruses (e.g., retroviruses and influenza viruses). In fact, recent evidence from a number of laboratories has 
confirmed the existence of numerous miRNAs in herpesviruses, has identified a single miRNA in adenovirus and SV40, and has so far failed to detect miRNAs in any RNA virus (Pfeffer et al. 2004, 2005; Cai et al. 2005, 2006; Grey et al. 2005; Sullivan et al. 2005; Grundhoff et al. 2006).

This review focuses on the miRNAs encoded by the gamma herpesvirus Kaposi's sarcoma-associated herpesvirus (KSHV), which is currently one of the best understood viruses in terms of viral miRNA expression and function. This research, together with analogous efforts focusing on a range of other pathogenic herpesviruses, suggests that miRNAs are likely to have a key role in mediating the ability of herpesviruses to maintain long-term latent infections in vivo in the face of host adaptive and innate immune responses.

\section{KSHV EXPRESSES 12 MICRORNAS IN LATENTLY INFECTED CELLS}

KSHV readily establishes latent infections in human B cells, where the virus then maintains 50-100 episomal copies of its circular viral DNA genome (Chen and Lagunoff 2005). Although KSHV encodes more than 80 proteins in its approximately 140,000-bp genome, only a small subset of these are expressed in latently infected cells (Russo et al. 1996; Sarid et al. 1998; Jenner et al. 2001). These minimally include ORF73 (LANA), which has a key role in the maintenance of the viral episome (Ye et al. 2004); ORF72 (v-cyclin), which is thought to regulate cell cycle progression in infected cells; ORF71 (VFLIP), an antiapoptotic protein; and finally Kaposin, which appears to have oncogenic properties (McCormick and Ganem 2005). Of note, the genes encoding these four proteins are clustered together in the "latency-associated region" of the KSHV genome (Fig. 1).

On the basis of the hypothesis that viral miRNAs might be particularly advantageous to DNA viruses during latent infection (Cullen 2006), we cDNA-cloned candidate miRNAs from the latently KSHV-infected cell line BC-1 by size selection for RNAs that were greater than 18 nucleotides and less than 25 nucleotides in size (Cai et al. 2005). This identified 167 miRNA cDNA clones of KSHV origin, and these could all be assigned to ten different miRNA stem-loop precursors designated miR-K1 to miR-K10 (Fig. 2). Work from other investigators subsequently identified two more KSHV miRNAs, miR-K11 and miR-K12, that were missed in our original analysis (Pfeffer et al. 2005; Samols et al. 2005; Grundhoff et al. 2006). All 12 KSHV miRNAs form part of one arm of an approximately 80-nucleotide RNA hairpin structure, and in several cases, the predicted "passenger" strand, that is, the strand of the miRNA duplex intermediate that is normally excluded from RISC and degraded, was also recovered (Fig. 2). This allowed confirmation of the expectation that the KSHV miRNA duplex intermediates would contain the 2-nucleotide $3^{\prime}$ overhangs that arise from sequential processing by Drosha and Dicer.

A surprising observation to emerge from these studies was the clustering of all $12 \mathrm{KSHV}$ miRNAs into an approximately $4-\mathrm{kb}$ region of the approximately $140-\mathrm{kb}$ KSHV genome that coincides with the KSHV "latency associated region." Specifically, 10 of the KSHV miRNAs mapped to a noncoding region located between the Kaposin and ORF71 genes (Fig. 1); one miRNA, miR-K10, was actually located within an open reading frame (ORF) that forms part of the Kaposin gene, whereas the last miRNA, miR-K12, mapped to the $3^{\prime}-$ untranslated region (3'UTR) of Kaposin (Fig. 1). Importantly, all 12 miRNAs were found to be in the same transcriptional orientation, thus suggesting that they might all be processed out of a single pri-miRNA precursor. Several cellular miRNA “clusters" have been previously identified (Bartel 2004), so this would certainly not be unprecedented.
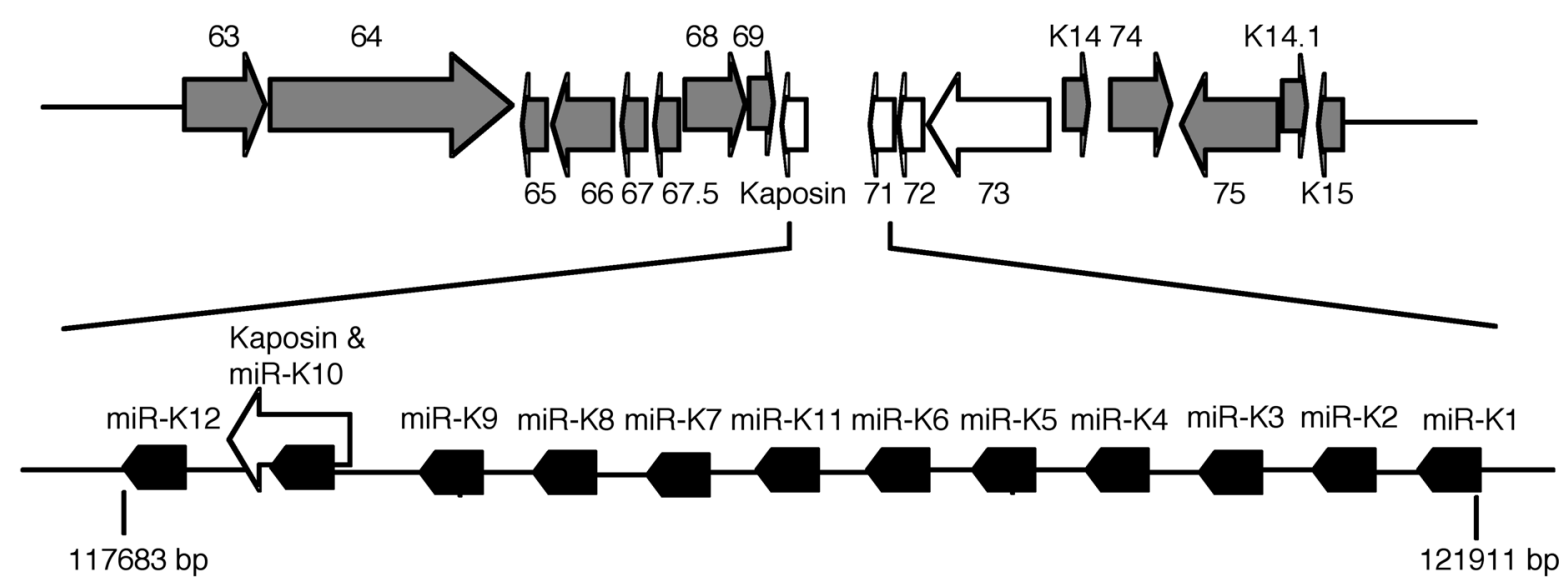

Figure 1. Genomic location of KSHV miRNAs. Schematic of a region of the KSHV genome. Known protein-coding genes, their orientation, and expression patterns are indicated. Ten of the KSHV miRNAs are arrayed between the ORF71 and Kaposin genes, one (miR-K10) overlaps the Kaposin ORF, whereas miR-K12 is found in the Kaposin 3'UTR. (White boxes) KSHV genes expressed during latent infection; (gray boxes) KSHV genes expressed during lytic infection; (black boxes) KSHV pri-miRNA precursors. The transcriptional orientation of each gene and each pri-miRNA hairpin is indicated. (Modified, with permission, from Cai et al. 2005 [C National Academy of Sciences].) 
Figure 2. Predicted stem-loop structures adopted by KSHV pri-miRNAs. The miRNA sequences identified by cDNA cloning are

highlighted. (Modified, with permission, from Cai et al. 2005 [C National Academy of Sciences].)
$\mathrm{miR}-\mathrm{K} 7$

$$
\begin{aligned}
& \text { U GU C ACG U CUGUAU } \\
& \begin{array}{llll} 
& \\
\text { GCAUAU GGC UGAGCGCCA CGG GGGAUU AUG } & \text { CCG ACUCGCGG GUU CCCUAG UAC }
\end{array} \\
& \text { C GC C GUA - CAUCAUU }
\end{aligned}
$$

miR-K8

$$
\begin{aligned}
& \text { 5' GCGC ACGCGCG CUC CUCA CUAA CC UUUGUCU } \\
& \text { CGCG CGCGCGC GAG GAGU GCGG CGA G } \\
& \text { G AC A CAGC AU CGAAGGUU }
\end{aligned}
$$

miR-K9

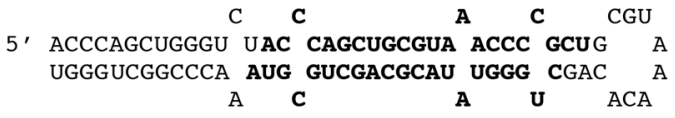

miR-K10

$$
\begin{aligned}
& \text { 5' GGGU GCGCUgG GCUUGgGg GAUA CAC UCG CAC C }
\end{aligned}
$$

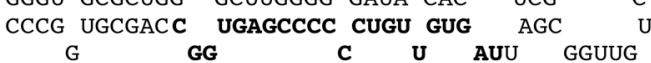

miR-K11

$$
\begin{aligned}
& \text { 5' }{ }^{\text {A }}{ }^{\circ} \text { UU } \mathrm{U} \text { CGC UUGG CACAG UUAA CAUU UCU GCGGUG } \\
& \text { UGC CC GCG AGCC GUGUC GAUU GUAA UCC U } \\
& \text { - CG U U C C U-- UAGUAU }
\end{aligned}
$$

miR-K12

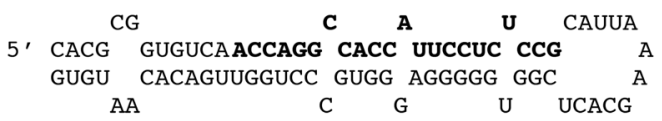

Because the latency-associated region of the KSHV genome is the only region that has been found to be consistently transcribed in all latently KSHV-infected cells (Dittmer et al. 1998; Sarid et al. 1998; Jenner et al. 2001), and because KSHV infection can induce tumorigenesis in infected immunosuppressed individuals, there has been considerable interest in the transcriptional regulation of the four genes found in this region of the KSHV genome. Building on this important earlier research (Dittmer et al. 1998; Li et al. 2002; Pearce et al. 2005), we performed a detailed analysis of the RNA transcripts that arise from this region, focusing particularly on RNAs that would be predicted to function as viral pri-miRNAs (Cai et al. 2006). This analysis was performed both in latently KSHV-infected B cells and in cells that had been induced to enter the lytic viral replication cycle by treatment with TPA.

In latently KSHV-infected B cells, we mapped two transcriptionally active promoters with imprecise cap sites located at 127,880 to 127,886 and 123,751 to 123,760 , respectively (Fig. 3). The promoter located at about 127,880 was found to give rise to four distinct mRNAs, depending on which polyadenylation (pA) site and which splice sites are utilized (Cai et al. 2006). If the
pA site at 122,070 is used, then transcription from the 127,880 promoter gives rise to two spliced mRNAs that have the potential to encode the viral proteins ORF71, ORF72, and ORF73. Neither of these two mRNAs has the potential to function as a pri-miRNA. However, if the pA site at 122,070 is bypassed, which occurs about $50 \%$ of the time, then transcripts initiating at the 127,880 promoter are instead polyadenylated at the efficient PA site located at 117,436 (Fig. 3). These pre-mRNA transcripts are spliced such that the ORF71-, 72-, and 73-coding sequences are removed and the resultant mature mRNAs instead express Kaposin (Fig. 3). Importantly, the KSHV miRNAs miR-K1 to miR-K9, as well as miR-K11, are all located within the intron(s) that is spliced out of these Kaposin mRNAs. As noted above, many cellular miRNAs are also found within introns. In work published elsewhere (Cai et al. 2006), we were able to directly confirm that these KSHV miRNAs can indeed be processed out of the introns present in the Kaposin pre-mRNAs that are transcribed from the 127,880 promoter.

In addition to the 127,880 promoter, KSHV also contains a second, slightly less active, latent promoter that initiates transcription at $123,751 / 123,760$. This promoter can again give rise to two very different mRNAs depending on whether the pA site located at 122,070 is used or ignored. If it is used, then an unspliced approximately 1.7-kb mRNA encoding ORF71 and ORF72 is expressed. 

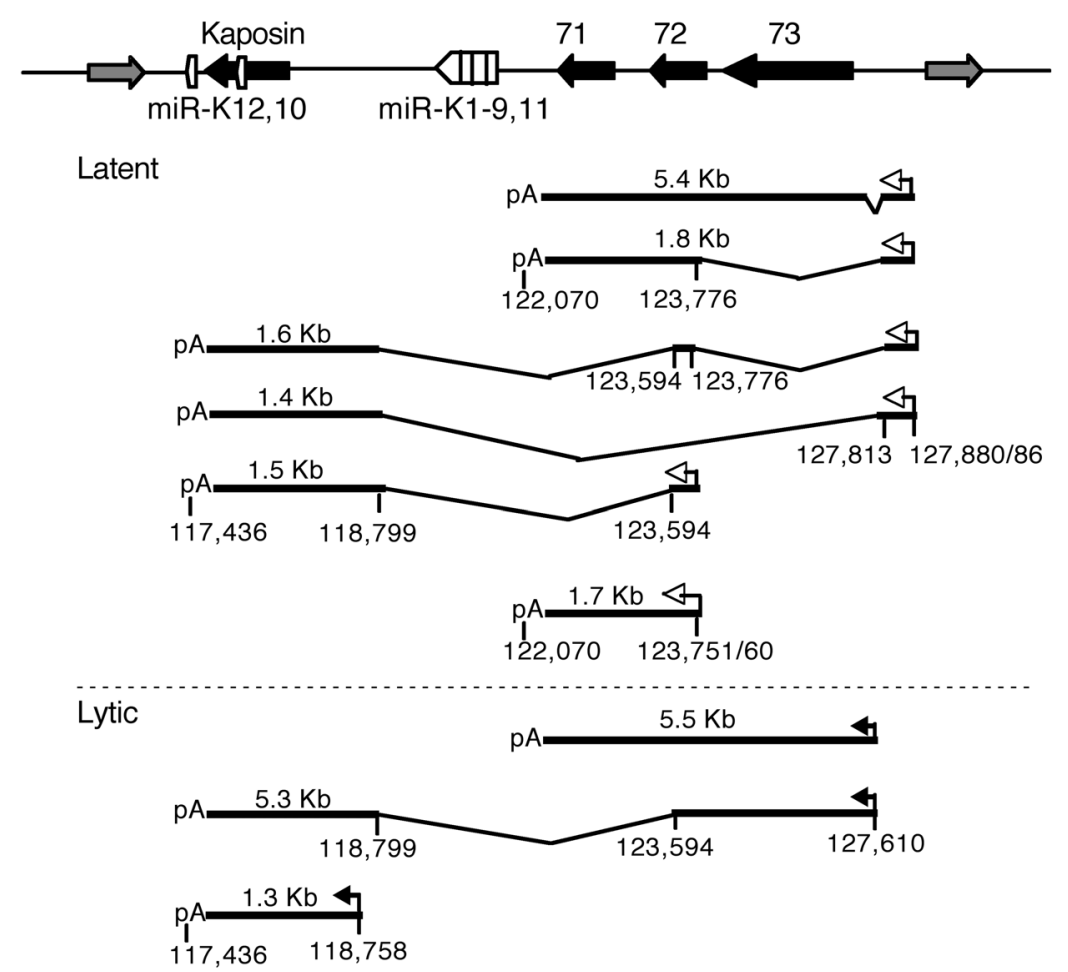

Figure 3. Transcript map of the latency-associated region of the KSHV genome. Shown is a schematic of the genes and miRNAs found in the KSHV latency-associated region as well as the pre-mRNAs and mature mRNAs that have been identified. Numbers refer to the sequence coordinates of transcription start sites, splice sites, and polyadenylation sites within the KSHV genome. Latent transcripts are shown above the dashed line and lytic transcripts below the line. Sizes of mature mRNAs are given in kilobases, and their promoters are indicated by arrows. (pA) Poly(A)-addition site. (Modified, with permission, from Cai and Cullen 2006 [C ASM].)

If it is ignored, then an approximately $1.5-\mathrm{kb}$ spliced mRNA encoding Kaposin is expressed. This latter mRNA again has the potential to function as a primiRNA for all 12 KSHV miRNAs (Fig. 3).

Although miR-K1 to miR-K9, as well as miR-K11, are all located in the intron(s) excised from the Kaposin miRNAs described in Figure 3, two miRNAs, miR-K10 and miR-K12, are located in the Kaposin ORF and 3’UTR, respectively (see Fig. 1). As noted above, excision of either of these miRNAs in the nucleus of latently KSHV-infected cells would appear to be inconsistent with mRNA function and hence with the expression of the Kaposin proteins. Two alternative hypotheses can be advanced to explain this apparent contradiction. One possibility is that processing of the miR-K10 and miR-K12 pri-miRNAs by the microprocessor complex is inefficient, so that a substantial percentage of Kaposin mRNA is able to exit the nucleus in an intact form. Alternatively, it is possible that KSHV is able to regulate pri-miRNA processing in some way, such that miR$\mathrm{K} 10$ and miR-K12 expression is only activated when necessary.

We considered the possibility that miR-K10 and miR$\mathrm{K} 12$ are actually not overly important during latent infection and only become critical during lytic KSHV replication. To address this hypothesis, we sought to identify promoters located within the KSHV latencyassociated region that are activated during lytic replication. It is important to note that the two latent promoters located in this region are neither activated nor inhibited by induction of lytic KSHV replication (Dittmer et al. 1998; Cai et al. 2006).

As shown in Figure 3, we and other investigators have identified two lytic promoters in this region of the KSHV genome. One of these promoters, with a cap site located at 118,758 , is an extremely powerful promoter that gives rise to high levels of about $1.3 \mathrm{~kb}$ that is predicted to encode Kaposin and also act as a pri-miRNA for miRK10 and miR-K12 only (Sadler et al. 1999; Cai et al. 2006). A second lytic promoter, with a cap site located at 127,610 , is a relatively weak promoter (comparable in strength to the latent 127,880 promoter) and is predicted to give rise to two mature mRNAs, again depending on whether the pA site at 122,070 is used or not (Cai et al. 2006). These are an unspliced approximately $5.5-\mathrm{kb}$ mRNA potentially encoding ORF71, 72, and 73 and an approximately 5.3-kb mRNA encoding Kaposin and all 12 KSHV miRNAs.

Because the KSHV lytic 127,610 promoter is comparable in activity to the latent promoters located at 127,880 and 123,751 , one would predict that induction of lytic replication would only modestly enhance the expression of the KSHV miR-K1 to miR-K9 and miR-K11 miRNAs. In contrast, because the KSHV lytic 118,758 promoter is much more active than these two latent promoters (Cai et al. 2006), one would predict that induction of lytic replication would strongly activate the expression of miR-K10 and miR-K12. As shown in Figure 4, this is 


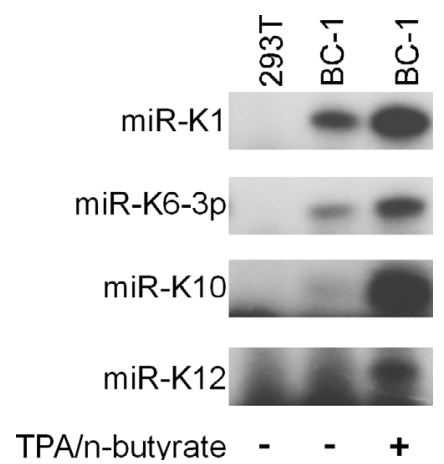

Figure 4. Effect of induction of lytic KSHV replication on viral miRNA expression in $\mathrm{BC}-1$ cells. $\mathrm{BC}-1$ cells were either cultured normally or treated with TPA $(30 \mathrm{ng} / \mathrm{ml})$ and $n$-butyrate (300 ng/ml) for 48 hours prior to RNA isolation as described previously (Cai and Cullen 2006). The level of expression of the indicated KSHV miRNAs was then determined by primer extension analysis (Cai and Cullen 2006). Uninfected 293T cells were used as the negative control.

indeed the case. Specifically, induction of lytic KSHV replication by treatment of BC-1 cells with TPA and $n$ butyrate enhanced the expression of miR-K1 and miRK6-3p by two- to threefold but had a far more dramatic effect on the expression of miR-K10 and miR-K12, which are actually quite difficult to detect in latently infected cells. These observations are consistent with the hypothesis that processing of miR-K10 and miR-K12 by Drosha is inefficient, so that high-level expression of these two viral miRNAs is only achieved when high-level expression of the underlying Kaposin mRNA is induced. However, it remains possible that miRNA processing efficiency, either in general or of these two viral miRNAs in particular, is somehow enhanced upon activation of lytic KSHV replication.

\section{KSHV MIRNAS ARE BIOLOGICALLY ACTIVE}

As noted above, miRNAs function as guide RNAs for RISC. If they successfully direct RISC to an mRNA bearing a highly complementary target site, then it is expected that that mRNA will be cleaved by RISC and subsequently degraded by the action of cellular exonucleases (Bartel 2004).

To confirm that the KSHV miRNAs are indeed programming active RISC complexes in latently KSHVinfected cells, we constructed a set of two lentivirus-based indicator vectors containing, respectively, a renilla luciferase (RLuc) or a firefly luciferase (FLuc) indicator gene (Fig. 5, top) (Gottwein et al. 2006). Moreover, the RLuc indicator virus was engineered to contain two tandem target sites in its $3^{\prime}$ UTR that are perfectly complementary to one of the KSHV miRNAs-miR-K1 to miR-K11 (Fig. 5, top). In the case of miR-K4, we constructed indicator viruses specific to KSHV miRNAs derived from both the $5^{\prime}$ arm (miR-K4-5p) and the $3^{\prime}$ arm (miR-K4-3p) of the pre-miRNA precursor as both were cDNA-cloned from latently KSHV-infected cells (Cai et al. 2005).

To confirm that the KSHV miRNAs are indeed biologically active, we prepared a viral stock of the FLuc-based control virus and a viral stock for each of the 13 RLucbased indicator viruses by transfection of $293 \mathrm{~T}$ cells. The FLuc viral stock was then mixed with each of the RLuc viral stocks, and the resultant mixture was used to infect
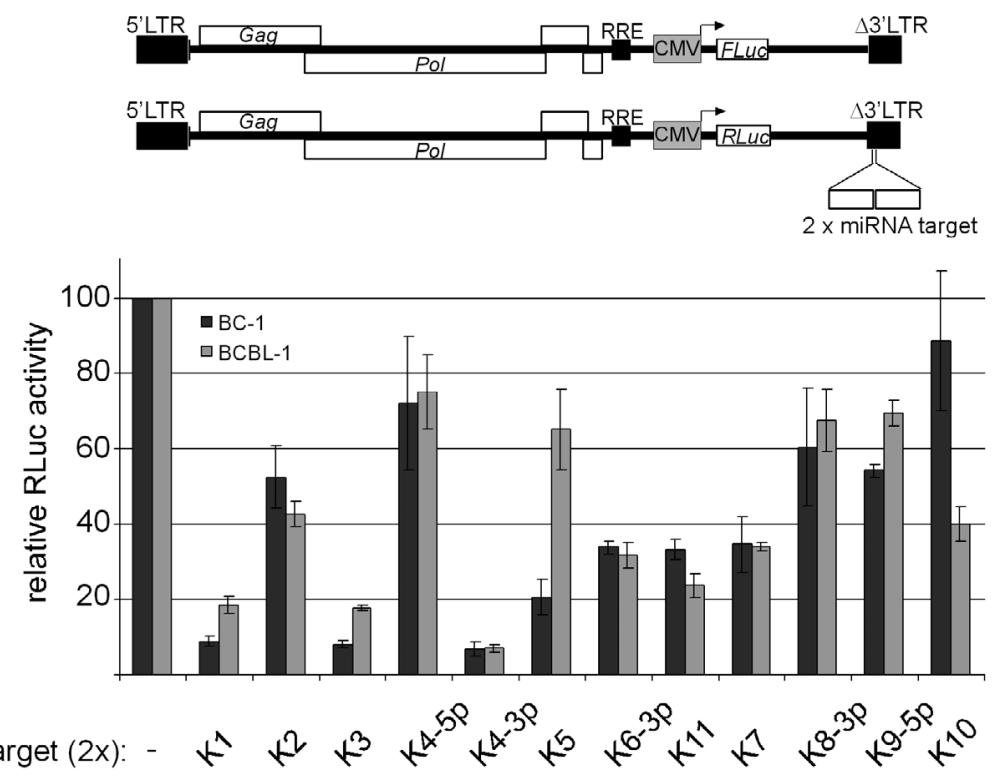

Figure 5. (Top) Schematic of the control vector pNL-SIN-CMV-FL, which encodes FLuc, and the indicator vector pNL-SIN-CMV$\mathrm{RL}$, which encodes RLuc. In each RLuc-based indicator construct, two perfectly complementary target sequences for one KSHV miRNA were inserted into the 3'UTR of the Renilla luciferase gene. (Bottom) BJAB, BC-1, and BCBL-1 cells were transduced with a mixture of the control and indicator viruses, and dual luciferase assays were performed. Normalized RLuc activities in KSHVpositive BC-1 and BCBL-1 cells are shown relative to those obtained in KSHV-negative BJAB cells. Values from cells transduced with the parental RLuc-expressing vector were set at 100\%. (LTR) Long terminal repeat; (RRE) Rev response element; (CMV) cytomegalovirus immediate-early promoter. (Modified, with permission, from Gottwein et al. 2006 [C ASM].) 
the latently KSHV-infected B-cell lines BC-1 and BCBL-1, as well as the uninfected human B-cell line BJAB. The level of RLuc and FLuc activity was then determined at 24 hours after transduction (Fig. 5, bottom). The data are presented relative to the level of RLuc activity seen in the uninfected BJAB cell line, which was set at 100 , after correction for variations in the level of activity seen with the FLuc internal control indicator virus (Gottwein et al. 2006). As may be observed (Fig. 5, bottom), all the KSHV miRNAs inhibited RLuc expression to some degree. The inhibition observed was profound with miR$\mathrm{K} 1$, miR-K3, and miR-K4-3p, modest with miR-K2, miR-K6-3p, miR-K11, and miR-K7, and fairly weak with miR-K8-3p, miR-K9-5p, and miR-K10.

One interesting observation was that the level of inhibition was consistent between the two KSHV-infected cell lines tested, that is, BC-1 and BCBL-1, with the exception of miR-K5 and possibly miR-K10. Analysis of the level of expression of the mature KSHV miR-K5 miRNA and of the pre-miR-K5 intermediate in fact revealed that both are expressed at much lower levels in BCBL-1 cells, where miR-K5 is less active, than in BC-1 cells (Fig. 6, top left). In contrast, all other KSHV miRNAs, including miR-K10, are expressed at comparable levels in the two cell lines (Fig. 6, top left) (Cai et al. 2005).

To try to understand the molecular mechanism underlying this discrepancy, we first sequenced the pri-miR-K5 stem-loop precursor in both BC-1 and BCBL-1 cells. As shown in Figure 6 (top right), this analysis revealed a single nucleotide polymorphism $(\mathrm{G} \rightarrow \mathrm{A})$ in BCBL-1 relative to $\mathrm{BC}-1$ that is predicted to disrupt a $\mathrm{G}$ : $\mathrm{C}$ base pair in the pri-miR-K5 transcript. Of note, this change is in the passenger strand, not in the mature miR-K5 miRNA itself. We and other investigators have previously shown that efficient processing of a pri-miRNA precursor to a mature miRNA requires the entire miRNA stem-loop structure, including the basal extension of the stem that is excluded from the pre-miRNA hairpin intermediate, as well as a small amount (generally 20 nucleotides or so) of flanking single-stranded RNA sequence (Lee et al. 2003; Zeng and Cullen 2003).

To test whether this one nucleotide polymorphism in fact accounted for the poor expression of miR-K5 in BCBL-1 cells, we used PCR (polymerase chain reaction) to clone analogous 472-bp segments of the KSHV genome, centered on the pri-miR-K5 stem-loop, from both BC-1 and BCBL-1 cells and inserted these into the tetracycline-regulated $\mathrm{pTre}$ expression vector. These two matched KSHV miRNA expression vectors were then transfected into $293 \mathrm{~T}$ cells, in the presence or absence of the pTet-Off plasmid and doxycycline. As expected, transfection of 293T cells with pTre-miR-K5(BC-1), in the presence of pTet-Off and the absence of doxycycline, gave rise to readily detectable levels of "pri-miR-K5," pre-miR-K5, and mature miR-K5. In contrast, in cells transfected with the very similar pTre-miR-K5(BCBL-1)

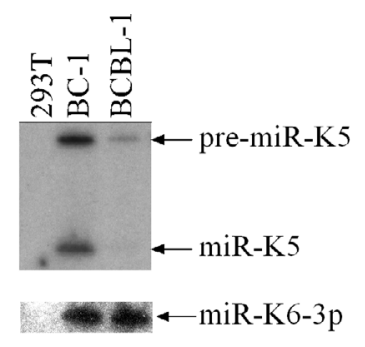

\section{miR-K5(BC-1)}
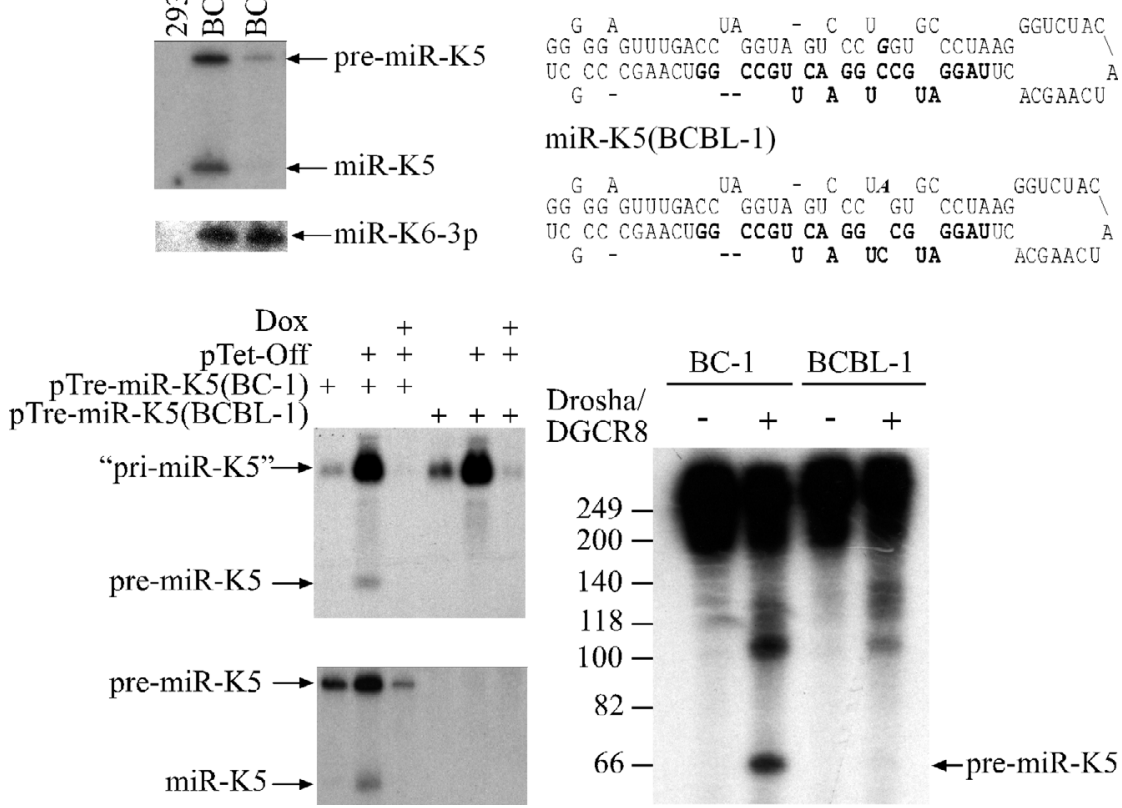

Figure 6. Differential expression of miR-K5 in BC-1 and BCBL-1 cells. (Top left) Northern analysis of miR-K5 (upper panel) and miR-K6-3p expression in total RNA preparations from 293T, BC-1, or BCBL-1 cells. (Top right) Predicted pre-miR-K5 stem-loop structures encoded by BC-1-derived (upper panel) or BCBL-1-derived (lower panel) KSHV. The mature miR-K5 sequence is shown in bold, and the single-nucleotide difference is shown in bold and italics. (Bottom left) Northern analysis of total RNA preparations from $293 \mathrm{~T}$ cells transfected with the indicated plasmids and cultured in the presence or absence of doxycycline (Dox). The miR-K5 pri-miRNA and pre-miRNA precursors as well as mature miR-K5 are indicated. The quotation marks flanking "pri-miR-K5" signify that this RNA represents a truncated form of the authentic viral pri-miR-K5 transcript. (Bottom right) BC-1- or BCBL-1-derived primiR-K5 transcripts were incubated in vitro with recombinant Flag-Drosha/Flag-DGCR-8(+) or with control extracts prepared $(-)$. The processed pre-miRNA is indicated by an arrow. (Modified, with permission, from Gottwein et al. 2006 [C ASM].) 
vector, only the artificial pri-miR-K5 transcript was detected (Fig. 6, bottom left).

The data presented in Figure 6 (bottom left) suggested, but did not prove, that the one nucleotide polymorphism identified in Figure 6 (top right) inhibited pri-miR-K5 processing by the nuclear microprocessor complex. To confirm that this was indeed the case, we overexpressed Flag-tagged forms of human Drosha and DGCR8 in 293T cells and then recovered the recombinant Drosha-DGCR8 complex from these cells using an affinity matrix (Gottwein et al. 2006). This matrix was then incubated with a 270 -nucleotide ${ }^{32} \mathrm{P}$-labeled transcript encompassing the entire KSHV pri-miR-K5 stem-loop and adjacent flanking sequences derived from either BC-1 or BCBL1 cells. As may be observed (Fig. 6, bottom right), the recombinant Drosha-DGCR8 complex cleaved the RNA transcript derived from $\mathrm{BC}-1$ cells to give the predicted 62-nucleotide pri-miR-K5 intermediate, as well as the two approximately 100-nucleotide flanking RNA sequences. In contrast, very little Drosha processing of the analogous pri-miR-K5 RNA template derived from BCBL-1 cells was observed. We therefore conclude that the weak activity (Fig. 5, bottom) and low level of expression (Fig. 6, top left) of miR-K5 seen in BCBL-1 cells result from a single-nucleotide polymorphism (Fig. 6 , top right) that inhibits processing of the pri-miR-K5 precursor by Drosha (Fig. 6, bottom right). To our knowledge, this is the first demonstration of a naturally occurring sequence polymorphism that directly perturbs miRNA processing and hence function.

\section{CONCLUSIONS}

This paper has summarized our current understanding of the expression and function of the miRNAs encoded by the pathogenic human herpesvirus KSHV. Work from our laboratory and others has identified at least 12 distinct miRNAs in the KSHV genome (Cai et al. 2005; Pfeffer et al. 2005; Grundhoff et al. 2006), all of which are expressed in latently infected cells (Fig. 2). Surprisingly, these 12 miRNAs proved to be clustered together in an approximately $4-\mathrm{kb}$ region of the approximately $140-\mathrm{kb}$ KSHV genome that coincided with the previously defined KSHV latency-associated region (Fig. 1) (Dittmer et al. 1998). Moreover, these 12 miRNAs were all in the same transcriptional orientation, thus suggesting that they might all derive from a single pri-miRNA precursor. In latently infected cells, this has indeed proven to be the case (Fig. 3) (Cai et al. 2006). However, in lytically infected cells, activation of a lytic promoter that is normally silent during latent infection gives rise to a distinct and very highly expressed transcript that functions both as a pri-miRNA precursor for two of the KSHV miRNAs, that is, miR-K10 and miR-K12, and as an mRNA encoding the viral Kaposin proteins (Sadler et al. 1999; Cai et al. 2006). This is an unexpected result, as miRNA processing results in the nuclear degradation of the pri-miRNA precursor so that it cannot also function as an mRNA. The most likely explanation for this paradox is that miR-K10 and miR$\mathrm{K} 12$ processing is very inefficient, so that much of the Kaposin mRNA is able to reach the cytoplasm intact.
Indeed, expression of miR-K10 and miR-K12 in latently infected cells appears to be quite low, in comparison to other KSHV miRNAs, even though they all derive from processing of the same latent transcripts (Fig. 4).

The obvious and most important questions with regard to the miRNAs encoded by KSHV and other herpesviruses are, What are their function in the viral life cycle, and what are their mRNA targets? At present, the answers to these questions are not known. However, the fact that ten of the KSHV miRNAs are expressed at readily detectable and biologically active levels in latently infected cells (Fig. 5) whereas two KSHV miRNAs only appear to become fully active during lytic replication (Fig. 4) suggests that these miRNAs may have distinct roles during the viral life cycle.

\section{ACKNOWLEDGMENTS}

This research was funded by grant GM071408 from the National Institutes of Health. The authors thank Blossom Damania and Shihua Lu for their help with aspects of this research.

\section{REFERENCES}

Bartel D.P. 2004. MicroRNAs: Genomics, biogenesis, mechanism, and function. Cell 116: 281.

Cai X. and Cullen B.R. 2006. Transcriptional origin of Kaposi's sarcoma-associated herpesvirus microRNAs. J. Virol. 80: 2234.

Cai X., Hagedorn C.H., and Cullen B.R. 2004. Human microRNAs are processed from capped, polyadenylated transcripts that can also function as mRNAs. RNA 10: 1957.

Cai X., Lu S., Zhang Z., Gonzalez C.M., Damania B., and Cullen B.R. 2005. Kaposi's sarcoma-associated herpesvirus expresses an array of viral microRNAs in latently infected cells. Proc. Natl. Acad. Sci. 102: 5570.

Cai X., Schäfer A., Lu S., Bilello J.P., Desrosiers R.C., Edwards R., Raab-Traub N., and Cullen B.R. 2006. Epstein-Barr virus microRNAs are evolutionarily conserved and differentially expressed. PLoS Pathogens 2: e23.

Chen L. and Lagunoff M. 2005. Establishment and maintenance of Kaposi's sarcoma-associated herpesvirus latency in B cells. J. Virol. 79: 14383.

Cullen B.R. 2004. Transcription and processing of human microRNA precursors. Mol. Cell 16: 861 . . 2006. Viruses and microRNAs. Nat. Genet. 38: S25.

Denli A.M., Tops B.B.J., Plasterk R.H.A., Ketting R.F., and Hannon G.J. 2004. Processing of primary microRNAs by the microprocessor complex. Nature 432: 231.

Dittmer D., Lagunoff M., Renne R., Staskus K., Haase A., and Ganem D. 1998. A cluster of latently expressed genes in Kaposi's sarcoma-associated herpesvirus. J. Virol. 72: 8309.

Doench J.G., Petersen C.P., and Sharp P.A. 2003. siRNAs can function as miRNAs. Genes Dev. 17: 438.

Gottwein E., Cai X., and Cullen B.R. 2006. A novel assay for viral microRNA function identifies a single nucleotide polymorphism that affects Drosha processing. J. Virol. 80: 5321.

Gregory R.I., Yan K.-P., Amuthan G., Chendrimada T., Doratotaj B., Cooch N., and Shickhattar R. 2004. The microprocessor complex mediates the genesis of miRNAs. Nature 432: 235

Grey F., Antoniewicz A., Allen E., Saugstad J., McShea A., Carrington J. C., and Nelson J. 2005. Identification and characterization of human cytomegalovirus-encoded microRNAs. J. Virol. 79: 12095.

Grundhoff A., Sullivan C.S., and Ganem D. 2006. A combined computational and microarray-based approach identifies novel microRNAs encoded by human gamma-herpesviruses. RNA 12: 1 . 
Hammond S.M., Bernstein E., Beach D., and Hannon G.J. 2000. An RNA-directed nuclease mediates post-transcriptional gene silencing in Drosophila cells. Nature 404: 293.

Han J., Lee Y., Yeom K.-H., Kim Y.-K., Jin H., and Kim V.N. 2004. The Drosha-DGCR8 complex in primary microRNA processing. Genes Dev. 18: 3016.

Lee Y., Kim M., Han J., Yeom K.-H., Lee S., Baek S.H., and Kim V.N. 2004. MicroRNA genes are transcribed by RNA polymerase II. EMBO J. 23: 4051

Lee Y., Ahn C., Han J., Choi H., Kim J., Yim J., Lee J., Provost P., Radmark O., Kim S., and Kim V.N. 2003. The nuclear RNase III Drosha initiates microRNA processing. Nature 425: 415.

Li H., Komatsu T., Dezube B.J., and Kaye K.M. 2002. The Kaposi's sarcoma-associated herpesvirus K12 transcript from a primary effusion lymphoma contains complex repeat elements, is spliced, and initiates from a novel promoter. $J$. Virol. 76: 11880.

Martinez J., Patkaniowska A., Urlaub H., Lührmann R., and Tuschl T. 2002. Single-stranded antisense siRNAs guide target RNA cleavage in RNAi. Cell 110: 563.

McCormick C. and Ganem D. 2005. The Kaposin B protein of KSHV activates the p38/MK2 pathway and stabilizes cytokine mRNAs. Science 307: 739.

Pearce M., Matsumura S., and Wilson A.C. 2005. Transcripts encoding K12, v-FLIP, v-cyclin, and the microRNA cluster of Kaposi's sarcoma-associated herpesvirus originate from a common promoter. J. Virol. 79: 14457.

Pfeffer S., Zavolan M., Grässer F.A., Chien M., Russo J.J., Ju J., John B., Enright A.J., Marks D., Sander C., and Tuschl T. 2004. Identification of virus-encoded microRNAs. Science 304: 734 .

Pfeffer S., Sewer A., Lagos-Quintana M., Sheridan R., Sander C., Grässer F.A., van Dyk L.F., Ho C.K., Shuman S., Chien M., et al. 2005. Identification of microRNAs of the herpesvirus family. Nat. Methods 2: 269.

Jenner R.G., Alba M.M., Boshoff C., and Kellam P. 2001. Kaposi's sarcoma-associated herpesvirus latent and lytic gene expression as revealed by DNA arrays. J. Virol. 75: 891.
Russo J.J., Bohenzky R.A., Chien M.-C., Chen J., Yan M., Maddalena D., Parry J.P., Peruzzi D., Edelman I.S., Chang Y., and Moore P.S. 1996. Nucleotide sequence of the Kaposi sarcoma-associated herpesvirus (HHV8). Proc. Natl. Acad. Sci. 93: 14862.

Sadler R., Wu L., Forghani B., Renne R., Zhong W., Herndier B., and Ganem D. 1999. A complex translational program generates multiple novel proteins from the latently expressed Kaposin (K12) locus of Kaposi's sarcoma-associated herpesvirus. J. Virol. 73: 5722.

Samols M.A., Hu J., Skalsky R.L., and Renne R. 2005. Cloning and identification of a microRNA cluster within the latencyassociated region of Kaposi's sarcoma-associated herpesvirus. J. Virol. 79: 9301.

Sarid R., Flore O., Bohenzky R.A., Chang Y., and Moore P.S. 1998. Transcription mapping of the Kaposi's sarcomaassociated herpesvirus (human herpesvirus 8) genome in a body cavity-based lymphoma cell line (BC-1). J. Virol. 72: 1005.

Schwarz D.S., Hutvágner G., Haley B., and Zamore P.D. 2002. Evidence that siRNAs function as guides, not primers, in the Drosophila and human RNAi pathways. Mol. Cell 10: 537.

Sullivan C.S., Grundhoff A.T., Tevethia S., Pipas J.M., and Ganem D. 2005. SV40-encoded microRNAs regulate viral gene expression and reduce susceptibility to cytotoxic $\mathrm{T}$ cells. Nature 435: 682.

Ye F.-C., Zhou F.-C., Yoo S.M., Xie J.-P., Browning P.J., and Gao S.-J. 2004. Disruption of Kaposi's sarcoma-associated herpesvirus latent nuclear antigen leads to abortive episome persistence. J. Virol. 78: 11121.

Yi R., Qin Y., Macara I.G., and Cullen B.R. 2003. Exportin-5 mediates the nuclear export of pre-microRNAs and short hairpin RNAs. Genes Dev. 17: 3011.

Zeng Y. and Cullen B.R. 2003. Sequence requirements for micro RNA processing and function in human cells. RNA 9: 112.

Zeng Y., Yi R., and Cullen B.R. 2003. MicroRNAs and small interfering RNAs can inhibit mRNA expression by similar mechanisms. Proc. Natl. Acad. Sci. 100: 9779. 


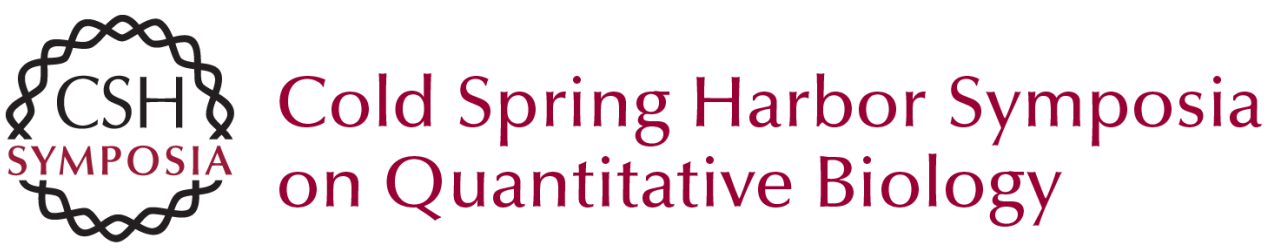

\section{Expression and Function of MicroRNAs Encoded by Kaposi's Sarcoma-associated Herpesvirus}

E. GOTTWEIN, X. CAI and B.R. CULLEN

Cold Spring Harb Symp Quant Biol 2006 71: 357-364

Access the most recent version at doi:10.1101/sqb.2006.71.004

References This article cites 36 articles, 24 of which can be accessed free at: http://symposium.cshlp.org/content/71/357.full.html\#ref-list-1

License

Email Alerting Receive free email alerts when new articles cite this article - sign up in Service the box at the top right corner of the article or click here. 